ISSN 0103-5150

Fisioter. Mov., Curitiba, v. 24, n. 3, p. 471-479, jul./set. 2011 Licenciado sob uma Licença Creative Commons

\title{
Análise dos efeitos do ultrassom terapêutico e da eletrolipoforese nas alterações decorrentes do fibroedema geloide
}

\author{
Analysis of the effects of the therapeutic ultrasound and the \\ electrolipophoresis in the current alterations of gynoid lipodistrophy
}

\author{
Giselle Cunha Machado $^{[a]}$, Rossana Bertolucci Vieira ${ }^{[b]}$, Nuno Miguel Lopes de Oliveira ${ }^{[c]}$, \\ Célia Regina Lopes $^{[\mathrm{d}]}$ \\ [a] Mestre, docente do Centro Universitário do Planalto de Araxá, Araxá, MG - Brasil, e-mail: giselle_c_m@hotmail.com \\ [b] Mestre, docente do Centro Universitário do Triângulo, Uberlândia, MG - Brasil, e-mail: rossanabv@hotmail.com \\ [c] Doutor, docente da Universidade Federal do Triângulo Mineiro, Uberaba, MG - Brasil, e-mail: pnmlo@hotmail.com \\ [d] Doutora, docente da Universidade Federal de Uberlândia, Uberlândia, MG - Brasil, e-mail: tsila80@hotmail.com
}

\section{Resumo}

Introdução: A síndrome da desarmonia corporal (SDC) inclui a presença de fibroedema geloide (FEG), adiposidade localizada, aumento de gordura corporal total e flacidez muscular - frequentemente associados - e esses distúrbios estéticos representam uma ameaça à integridade emocional do indivíduo, sendo uma variedade de terapias propostas para o seu tratamento. Objetivos: Avaliar os efeitos do ultrassom terapêutico (UST) e da eletrolipoforese no tratamento das alterações decorrentes do fibroedema geloide. Materiais e métodos: Foram estudadas 22 voluntárias, com idade entre 17-35 anos, presença de FEG graus 1 e/ou 2 em glúteos, sedentárias e usando anticoncepcional. Previamente foram avaliadas as variáveis de perimetria, sensibilidade, dor, satisfação pessoal, adipometria, avaliação fotográfica e bioimpedância elétrica bipolar. Foram realizadas 10 sessões com UST em 11 das voluntárias e as demais 11 voluntarias receberam tratamento por eletrolipoforese. Todas as técnicas foram aplicadas na região glútea bilateralmente, três vezes por semana, e ao término do tratamento as variáveis foram reavaliadas. $\mathrm{Na}$ análise estatística foi aplicado o teste $t$ de Student para verificar a significância entre os resultados obtidos em cada tratamento e entre os tratamentos. 0 nível de significância estabelecido foi de $\mathrm{p}<0,05$. Resultados: Não houve diferença estatisticamente significante na perimetria, adipometria e bioimpedância após os tratamentos instituídos. Na avaliação fotográfica houve melhora no aspecto visual do FEG em 68,18\% das participantes. A satisfação 
pessoal aumentou em ambos os grupos (UST $p=0,03^{*}$ e eletrolipólise $p=0,04^{*}$ ). Conclusão: Os tratamentos apresentaram melhora no aspecto visual do FEG e na satisfação pessoal, porém não alteraram medidas perimétricas, de adipometria e da bioimpedância.

Palavras-chave: Fisioterapia. Estética. Terapia por estimulação elétrica.

\begin{abstract}
Introduction: Body disharmony syndrome (CDS) includes the presence of fibroedema geloid (EGF), adiposity, increased total body fat and muscle weakness, often associated with these disorders and aesthetic pose a threat to the emotional integrity of the individual, where a variety therapies have been proposed for treatment. Objectives: To evaluate the effects of therapeutic ultrasound (TUS) and eletrolipoforese in the treatment of changes caused by fibroedema geloid. Materials and methods: We studied 22 volunteers, aged 17-35 years, presence of EGF and grades 1 or 2 in the buttocks, sedentary and contraceptive use. The variables were previously perimetry, sensitivity, pain, personal satisfaction, calipers, photographic assessment, bipolar electrical bioimpedance. Ten sessions were held with TU in 11 of the volunteers and the other 11 volunteers received treatment for eletrolipoforese. All techniques were applied bilaterally in the gluteal region, three times a week, and at the end of the treatment variables were evaluated. Statistical analysis was applied the Student t-test to verify the significance between the results obtained in each treatment and between treatments. Level of significance was set at $p<0.05$. Results: There were no statistically significant difference in girth, skinfold and BIA after their treatments. In the photographic evaluation was no improvement in visual appearance of EGF in $68.18 \%$ of the participants. Personal satisfaction increased in both groups ( $p=0.03 *$ UST eletrolipólise and $\left.p=0.04^{*}\right)$. Conclusion: The treatments showed an improvement in the visual aspect of the EGF and personal satisfaction, but not altered perimetric measures, calipers and bioelectrical impedance.
\end{abstract}

Keywords: Physical therapy. Esthetics. Electric stimulation therapy.

\section{Introdução}

A partir do conceito de saúde como completo bemestar físico, psíquico e social, e não apenas a ausência de doença, é possível compreender que o distúrbio estético representa uma ameaça à integridade emocional do indivíduo, resultante da alteração do esquema corporal e, consequentemente, da autoestima (1).

O conjunto de alguns dos problemas de beleza corporal é denominado síndrome da desarmonia corporal (SDC) e inclui a presença do fibroedema geloide (FEG), da adiposidade localizada, do aumento de gordura corporal total e da flacidez muscular, que frequentemente estão associados e, por isso, os tratamentos estéticos não devem considerar esses fenômenos isoladamente, a fim de promover ou manter a harmonia corporal (2).

No aspecto anátomo-histológico, o tecido com FEG encontra-se com aumento do número e do volume de células adiposas, lipoedema e dissociação lobular, espessamento e proliferação das fibras colágenas interadipocitárias e interlobulares, que provocam en- gurgitamento tecidual, rompimento das fibras elásticas, vasos linfáticos e sanguíneos ectásicos (3).

Estudos que analisaram a coxa de homens e mulheres por meio da ressonância magnética observaram diferença estrutural entre os gêneros, uma vez que nos homens o tecido adiposo é mais fino e apresenta uma rede de septos oblíquos e o tecido conetivo mais grosso, dividindo as câmaras gordurosas em pequenas unidades poligonais, enquanto nas mulheres notou-se grande porcentagem de septos, perpendiculares à derme, que separam os lóbulos adiposos volumosos em seções retangulares que pressionam a derme e geram o aspecto de casca de laranja visível na pele (4-6).

Essa forte rede de tecido conectivo na camada gordurosa e a derme mais grossa na pele masculina comparada à feminina podem prevenir as papilas adiposas de protrair em excesso a pele, enquanto a organização na mulher favorece a projeção do tecido gorduroso, formando irregularidades na pele. Isso poderia explicar por que o FEG afeta $90 \%$ das mulheres e, no caso dos homens, afeta poucos $(2,4,6)$. 
Tanto a variabilidade na estrutura do tecido conjuntivo como a susceptibilidade ao aparecimento do FEG estariam, principalmente, relacionados aos hormônios sexuais e não à genética do indivíduo, pois existem evidências de estruturas de fibras subcutâneas femininas na fáscia superficial de homens hipoandrogênicos $(6,7)$.

Além das diferenças quanto à anatomia do tecido adiposo, também o metabolismo dos adipócitos varia de região para região, onde o efeito lipolítico das catecolaminas é mais intenso e o efeito antilipolítico da insulina é menor nos depósitos gordurosos intra-abdominais, o que acarreta maior mobilização dos ácidos graxos livres nessa região comparada com a femoroglútea (8). Apesar dessa exacerbação do FEG com o ganho de peso, bem como a correlação ao índice de massa corporal, o FEG não deve ser confundido com obesidade (9-11).

Por ser uma desordem multifatorial, o tratamento do FEG deve contemplar diferentes aspectos e se direcionar a tratar a fibrose, a flacidez muscular, o acúmulo de gordura, a tonificação cutânea e o edema tecidual (12).

O FEG afeta milhões de mulheres em todo o mundo, porém só um limitado número de estudos referentes a esse assunto tem sido publicado na literatura científica, tendo muitos deles chegado a conclusões contraditórias (6). Uma variedade de terapias tem sido proposta para o tratamento do FEG objetivando a perda de peso, graças à diminuição da gordura subcutânea, reduzir a aparência da casca de laranja, o que leva à procura de métodos terapêuticos para redução de medidas de maneira mais eficaz (11).

A principal aplicação do UST envolve a produção de hiperemia, o aumento de leucócitos e anticorpos, a ação espasmolítica, a ação trófica, a analgesia, a antiflogística, o aumento da extensibilidade dos tendões, a destruição de macromoléculas, facilitar a reabsorção de edemas, a eliminação de macronódulos e do aspecto de casca de laranja no FEG, a correção da isquemia em áreas lipodistróficas, o aumento do intercâmbio iônico intercelular e a melhora do metabolismo lipídico com o aumento da lipólise (13).

A estimulação elétrica subcutânea gera redução de medidas, melhora do aspecto do FEG e a tonificação e rejuvenescimento de várias regiões do corpo (14). Tal estimulação tem efeitos nos tecidos excitáveis, gerando numerosos efeitos indiretos no organismo. Existem também evidências dos efeitos diretos que afetam o metabolismo dos tecidos (15), aumentando a circulação sanguínea local, estando relacionada ao aumento na geração de ATP pela célula $(14,16)$.

\section{Materiais e métodos}

O presente estudo refere-se a um ensaio clínico prospectivo randomizado, aprovado pelo Comitê de Ética em Pesquisa do Centro Universitário do Triângulo sob o Protocolo n. 635815, de acordo com a Resolução 196/96.

0 recrutamento das voluntárias foi realizado por meio de convite verbal às alunas dos diferentes cursos de graduação do Centro Universitário do Triângulo, onde foram recrutadas 78 voluntárias, das quais 48 estavam de acordo com os critérios de inclusão e exclusão da pesquisa.

Os critérios de inclusão foram: gênero feminino, faixa etária entre 18 e 35 anos, sedentárias, presença de FEG grau 1 e 2 em região glútea, tipo flácido, IMC entre 18 e $25 \mathrm{~kg} / \mathrm{m}^{2}$, uso de anticoncepcional e não estar em dieta alimentar. Os critérios para exclusão foram: tabagismo, diabetes, gravidez, indivíduos fazendo algum outro tipo de tratamento dermato-funcional, uso de medicamentos para emagrecimento, corticoides, progesterona e diuréticos, dificuldade em manter-se na posição ortostática, FEG grau 3 em região glútea, portadores de marca-passo e/ou doença cardíaca, tromboflebite, infecção aguda, áreas tratadas por radioterapia e tumores.

As voluntárias foram randomizadas em dois grupos de tratamento após serem informadas sobre o estudo e assinarem o Termo de Consentimento Livre e Esclarecido e o Termo de Autorização do Uso de Imagens, sendo: grupo I - ultrassom terapêutico (UST) e grupo II - eletrolipoforese. 0 tratamento proposto constituiu-se de uma avaliação pré-tratamento, 10 sessões de tratamento e uma última reavaliação pós-tratamento, totalizando 12 encontros.

Após avaliação prévia dos critérios de inclusão e exclusão, as voluntárias foram submetidas à avaliação inicial, seguindo o protocolo proposto por Meyer et al. (17), repetindo-o ao final do tratamento. A avaliação de todas as voluntárias foi composta respectivamente pela anamnese e pelo exame físico, constando dos seguintes itens:

- Inspeção e palpação: é realizada com o paciente na posição ortostática, uma vez que na posição 
de decúbito há acomodação dos tecidos, decorrente da ação da gravidade, podendo mascarar o grau de acometimento (18). A classificação do FEG foi feita, de acordo com os graus de classificação, em: I - visível só com contração muscular; II - visível sem compressão do tecido, acentuando-se com a contração; ou III tecido com grande número de relevos e depressões, apresentando macronódulos sensíveis à palpação, que foi analisado visualmente, sem contração muscular, e após com contração muscular na posição ortostática, sendo também feito o registro fotográfico digital para posterior avaliação. 0 teste de preensão quantificou a sensibilidade à dor em uma escala analógica de 0 a 10 pontos, sendo realizado por meio da preensão da pele com a tela subcutânea entre os dedos. Promove-se um movimento de tração e, quando a sensação dolorosa é mais incômoda que o normal, trata-se de um sinal de presença de FEG (18).

- IMC: foi definido pelo peso/altura ${ }^{2}$, considerando o critério da Organização Mundial da Saúde (OMS), determinado como: pacientes abaixo do peso ideal com IMC $<20 \mathrm{~kg} / \mathrm{m}^{2}$; dentro da faixa ideal de peso, com IMC entre 20 e $24,9 \mathrm{~kg} / \mathrm{m}^{2}$; com sobrepeso, IMC entre 25 e $29,9 \mathrm{~kg} / \mathrm{m}^{2}$; e obesidade, IMC acima de $30 \mathrm{~kg} / \mathrm{m}^{2}(19,20)$.

- Perimetria: é uma tradicional medida que indica a redução da circunferência da coxa pela redução do edema e do efeito na camada gordurosa (21). Com auxílio da fita métrica, foi feita a perimetria da coxa sendo demarcado o ponto de referência da linha poplítea e, a partir desse ponto, demarcados os outros pontos a cada 5 centímetros até a linha glútea. Foram ainda realizadas as medidas da cintura (cicatriz umbilical) e do quadril (na linha do trocanter maior) em posição ortostática e, posteriormente, foi calculada a relação cintura/ quadril (RCQ).

- Percentual de gordura: foi obtido a partir da técnica antropométrica, que se associa muito bem e não difere significativamente da pesagem hidrostática, que é tida como padrão ouro para avaliação de outras técnicas (19). As medidas foram realizadas no hemicorpo direito, com o paciente na posição ortostática com o membro relaxado, pinçando fortemente uma dobra de pele e gordura com o polegar e o indicador, destacando o tecido muscular e, a aproximadamente um centímetro abaixo da dobra, aplicando o adipômetro. Após 2 a 3 segundos realizou-se a leitura. Para se garantir maior confiabilidade nos dados, as mensurações foram executadas mais duas vezes, repetindo todos os procedimentos e anotando os dados. 0 valor médio de cada dobra foi utilizado como resultado (21). As dobras cutâneas avaliadas foram: tricipital, na dobra vertical, na face posterior do braço entre a borda lateral do acrômio e o olecrano; abdominal, na dobra vertical, situada a $2,5 \mathrm{~cm}$ à direita da cicatriz umbilical; e suprailíaca, verificada na dobra ligeiramente oblíqua, imediatamente acima da crista ilíaca. A soma desses valores foi utilizada para estimar a porcentagem de gordura de acordo com sexo e a idade, por meio da tabela Jackson e Pollock (18).

- bioimpedância elétrica bipolar: é um método rápido, não invasivo e de baixo custo, para avaliar a composição corporal, aplicado por um aparelho portátil, com intensidade de corrente de 500 a $800 \mathrm{~mA}$ e frequência de $50 \mathrm{KHz}$. A água corporal total é calculada e seu valor pode ser utilizado para estimar a gordura corporal (22). As voluntárias foram posicionadas em decúbito dorsal, os eletrodos autoadesivos foram posicionados nos membros superior e inferior do hemicorpo direito, na região dorsal de punho e pé, sendo o polo negativo próximo dos dedos e o positivo próximo do punho ou tornozelo. No aparelho foram inseridos: idade, altura, peso, sexo e nível de atividade da paciente e, assim, foi iniciado o processamento dos dados para a obtenção dos valores de gordura total, massa magra, água, taxa metabólica basal, IMC e impedância, em um ambiente controlado para evitar alterações.

- Registro fotográfico: foram realizadas fotografias da região glútea até a linha poplítea, com a paciente em posição ortostática, a máquina digital apoiada sobre um tripé a uma distância de dois metros das voluntárias e zoom de três vezes, antes e após o tratamento, com e sem contração muscular. As imagens foram analisadas pelo método duplo cego por dois profissionais experientes da área de fisioterapia dermato-funcional que quantificaram o 
grau de acometimento do FEG antes e após o tratamento selecionado, descrevendo qualitativamente a melhora do FEG.

- Escala de satisfação pessoal: a paciente classificava a sua satisfação pessoal, referente à sua harmonia corporal, em uma escala de 0 a 10 pontos, onde 0 foi considerado nenhuma satisfação e 10 a máxima satisfação (23).

Após a avaliação, as voluntárias foram subdivididas em dois grupos de tratamento, sendo 24 para o grupo I - UST (Ibramed ${ }^{\circledR}$ ) e 24 para o grupo II - eletrolipólise (GS1000 ${ }^{\circledR}$ ). Após a devida calibração dos aparelhos, as voluntárias foram posicionadas em decúbito ventral e, previamente ao início da terapia, a pele da paciente e o cabeçote ou os eletrodos dos aparelhos foram limpos com álcool. Foram realizadas dez sessões dos tratamentos propostos. Para a aplicação do UST, foi utilizado como meio de contato gel sem nenhum princípio ativo, o tratamento foi realizado bilateralmente dividindo-se a região glútea em quatro quadrantes. O UST foi usado no modo contínuo, na frequência de $3 \mathrm{MHz}$ e intensidade de $1,0 \mathrm{Wcm}^{2}$, com tempo de dois minutos para cada área de $10 \mathrm{~cm}(2)$.

Já para a aplicação da eletrolipoforese, foram utilizados quatro canais de saída de corrente, eletrodos de placa de silicone, contendo gel sem nenhum princípio ativo como meio de contato, e o programa determinado foi o de aplicação epicutânea, o qual é modulado da seguinte forma: 10: 5 minutos na frequência $50 \mathrm{Hertz}(\mathrm{Hz})$, tipo de onda $\mathrm{A}$ - retangular aguda; $2^{\circ}$ : 10 minutos na frequência $30 \mathrm{~Hz}$, tipo de onda B - retangular de base ampla; 3ㅇ: 20 minutos na freqüência $10 \mathrm{~Hz}$, tipo de onda $\mathrm{C}$ - trapezoidal aguda; e 4을 5 minutos na frequência $5 \mathrm{~Hz}$, tipo de onda D - trapezoidal de base ampla, totalizando 40 minutos de aplicação de corrente.

As placas foram fixadas na região posterior da coxa e no glúteo, bilateralmente e aos pares, intercalando o polo positivo com o negativo, paralelamente, da região interna até externa, sendo utilizados dois canais para cada hemicorpo, onde cada canal possui quatro eletrodos. A intensidade utilizada respeitou a sensibilidade e tolerância de cada paciente.

Durante o período de tratamento, foram excluídas da pesquisa 23 voluntárias, por abstinência, desistência e outros motivos que alterassem os resultados do estudo. A pesquisa foi concluída contendo apenas 11 voluntárias em cada um dos grupos de tratamento, conforme representado no esquema do Consort (Figura 1).

Para a análise estatística dos dados coletados inicialmente, foi realizado o teste de normalidade de Kolmogorov-Smirnov. Na sequência foi aplicado o teste de $t$ de Student para verificar a significância entre os resultados obtidos antes e após os tratamentos instituídos (Tabela 2) e para verificar se houve diferença estatisticamente significante entre os grupos de tratamento (Tabela 3 ). 0 coeficiente de concordância de Kendall (W) foi aplicado para verificar o nível de concordância entre os dois avaliadores das imagens fotográficas. 0 nível de significância foi estabelecido em $\mathrm{p}<0,05$.

\section{Resultados}

Nenhuma voluntária apresentou dor e alteração de sensibilidade aos testes realizados. Os dados antropométricos, como idade, peso, altura e IMC, demonstram a homogeneidade da amostra e estão representados na Tabela 1.

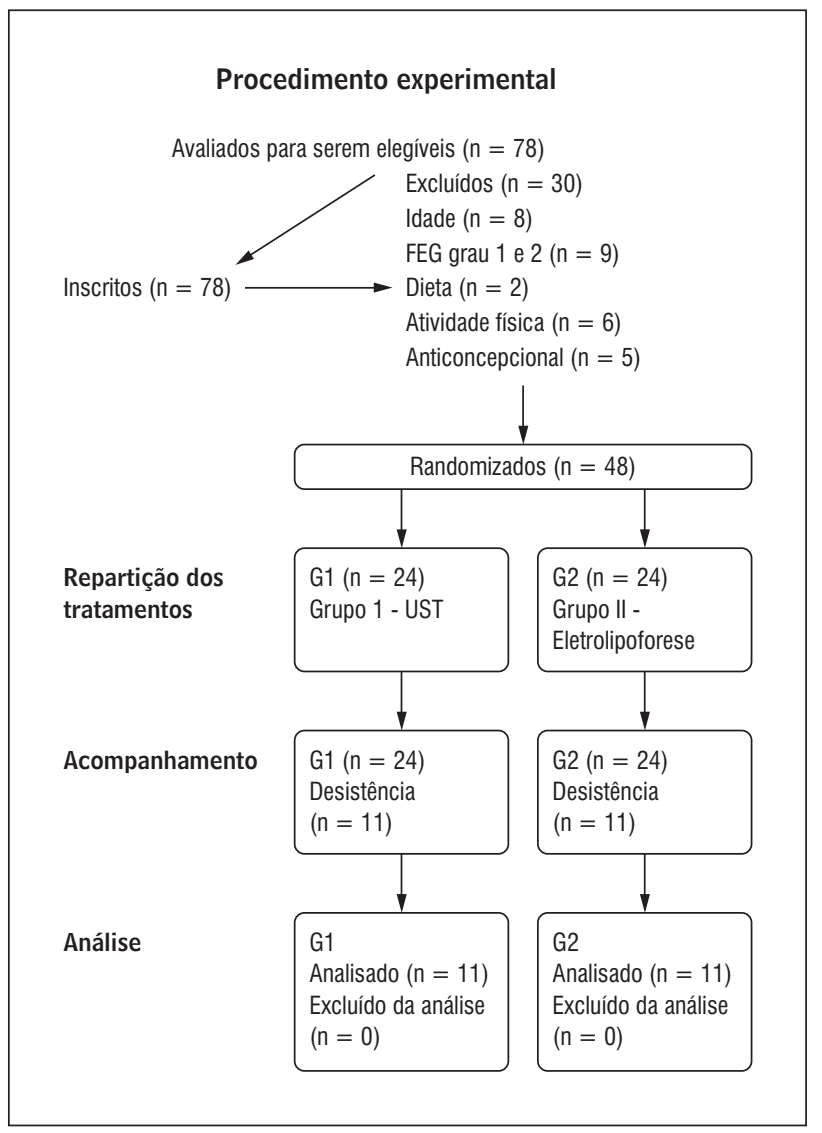

Figura 1 - Esquema do Consort 
Os resultados referentes aos valores de RCQ perimetria, dobras cutâneas e porcentagem de gordura avaliada pelas dobras cutâneas (\%Gord DC), analisando-se antes e após o tratamento eleito, não apresentaram diferença estatisticamente significante e estão demonstrados na Tabela 2.

Na Tabela 3 está representada a comparação das variáveis analisadas no estudo entre os dois diferentes grupos de tratamento sem diferença estatisticamente significante.

Houve diferença estatisticamente significante na análise da variável que considerou a satisfação pessoal das voluntárias (UST média: antes $=5( \pm 1) \mathrm{e}$ após $6( \pm 1)$, e mediana antes $=6$ e após $=6$; eletrolipoforese média: antes $=6( \pm 1)$ e após $7( \pm 2)$, e mediana antes $=4$ e após $=6$ ) considerando uma escala de 0 a 10 pontos antes e após o tratamento aplicado. 0 grupo UST apresentou valor de $p=0,03^{*}$ e o grupo eletrolipoforese, $\mathrm{p}=0,04^{*}$, ou seja, com melhora da satisfação pessoal em ambos os grupos, como representado na Figura 2.
Ao analisar as avaliações fotográficas realizadas pelos diferentes avaliadores, quantificando o acometimento do FEG antes e após o tratamento, verificou-se que $68,18 \%$ das participantes do grupo UST e $68,17 \%$ do grupo eletrolipólise apresentaram melhora do aspecto epitelial do fibroedema geloide. A concordância entre os dois avaliadores das imagens fotográficas foi elevada, obtendo valor de $\mathrm{W}>$ 0,65 para todas as imagens interpretadas.

Tabela 1 - Características antropométricas da amostra

\begin{tabular}{lccc}
\hline Variáveis & Ultrassom & Eletrolipoforese & N total = 22 \\
\hline Idade $($ anos) & $22( \pm 6)$ & $23( \pm 4)$ & $22( \pm 5)$ \\
Peso $(\mathrm{kg})$ & $55( \pm 4)$ & $56( \pm 7)$ & $55( \pm 5)$ \\
Altura $(\mathrm{m})$ & $1.63( \pm 0.04)$ & $1.63( \pm 0.07)$ & $1.63( \pm 0.06)$ \\
IMC $\left(\mathrm{k} / \mathrm{m}^{2}\right)$ & $21( \pm 2)$ & $21( \pm 2)$ & $21( \pm 2)$ \\
\hline
\end{tabular}

Legenda: Os valores representam a média ( \pm desvio-padrão). Fonte: Dados da pesquisa.

Tabela 2 - Comparação das medidas antropométricas e das medidas da bioimpedância antes e após as sessões de cada tratamento

\begin{tabular}{|c|c|c|c|c|c|c|}
\hline \multirow{2}{*}{$\begin{array}{l}\text { Variáveis } \\
\text { Média (DP) }\end{array}$} & \multicolumn{2}{|c|}{ Ultrassom terapêutico } & \multirow[b]{2}{*}{$\mathrm{p}$} & \multicolumn{2}{|c|}{ Eletrolipoforese } & \multirow[b]{2}{*}{$\mathrm{p}$} \\
\hline & Antes & Após & & Antes & Após & \\
\hline Cintura (cm) & $70( \pm 4)$ & $70( \pm 5)$ & 0.87 & $70( \pm 4)$ & $69( \pm 4)$ & 0.76 \\
\hline Quadril (cm) & $96( \pm 5)$ & $95( \pm 5)$ & 0.70 & $96( \pm 5)$ & $96( \pm 5)$ & 0.78 \\
\hline RCQ & $0.72( \pm 0.03)$ & $0.72( \pm 0.04)$ & 0.89 & $0.66( \pm 0.20)$ & $0.72( \pm 0.02)$ & 0.35 \\
\hline Superior coxa D & $53( \pm 4)$ & $53( \pm 0.04)$ & 0.90 & $53( \pm 3)$ & $52( \pm 3)$ & 0.37 \\
\hline Medial coxa D & $44( \pm 3)$ & $44( \pm 3)$ & 0.79 & $44( \pm 2)$ & $43( \pm 2)$ & 0.73 \\
\hline Inferior coxa D & $37( \pm 2)$ & $37( \pm 2)$ & 0.70 & $37( \pm 1)$ & $36( \pm 0)$ & 0.50 \\
\hline Superior coxa $\mathrm{E}$ & $53( \pm 4)$ & $53( \pm 4)$ & 0.95 & $53( \pm 4)$ & $52( \pm 3)$ & 0.43 \\
\hline Medial coxa E & $43( \pm 3)$ & $44( \pm 3)$ & 0.96 & $44( \pm 2)$ & $43( \pm 2)$ & 0.73 \\
\hline Inferior coxa $E$ & $37( \pm 2)$ & $37( \pm 2)$ & 0.99 & $37( \pm 2)$ & $37( \pm 2)$ & 0.67 \\
\hline +Dobras cutâneas & $47( \pm 17)$ & $47( \pm 17)$ & 0.95 & $53( \pm 10)$ & $50( \pm 10)$ & 0.57 \\
\hline$\%$ Gord DC & $21( \pm 5)$ & $22( \pm 5)$ & 0.94 & $23( \pm 3)$ & $23( \pm 3)$ & 0.50 \\
\hline Gordura (\%) & $25( \pm 4)$ & $24( \pm 3)$ & 0.72 & $25( \pm 3)$ & $26( \pm 3)$ & 0.90 \\
\hline M. magra (\%) & $42( \pm 4)$ & $43( \pm 4)$ & 0.71 & $42( \pm 6)$ & $42( \pm 5)$ & 0.91 \\
\hline Água (\%) & $53( \pm 3)$ & $53( \pm 3)$ & 0.78 & $53( \pm 3)$ & $53( \pm 3)$ & 0.78 \\
\hline Impedância (z) & $682( \pm 69)$ & $676( \pm 59)$ & 0.83 & $688( \pm 77)$ & $710( \pm 38)$ & 0.80 \\
\hline
\end{tabular}

Legenda: $\mathrm{DP}=$ desvio-padrão; $\mathrm{RCQ}=$ relação cintura/quadril; $\mathrm{D}=$ direita; $\mathrm{E}=$ esquerda; \% Gord $\mathrm{DC}=\%$ de gordura estimada pelas dobras cutâneas; * = valores significantes $p<0,05$.

Fonte: Dados da pesquisa. 
Tabela 3 - Comparação das medidas antropométricas e das medidas da bioimpedância entre os grupos de tratamento

\begin{tabular}{lccc}
\hline $\begin{array}{l}\text { Variáveis } \\
\text { pós-tratamento }\end{array}$ & $\begin{array}{c}\text { Ultrassom } \\
\text { Média (DP) }\end{array}$ & $\begin{array}{c}\text { Eletrolipoforese } \\
\text { Média (DP) }\end{array}$ & $\mathbf{p ~}$ \\
\hline Quadril (cm) & $95( \pm) 5$ & $96( \pm) 5$ & 0.80 \\
Superior coxa D & $53( \pm) 0.04$ & $52( \pm) 3$ & 0.37 \\
Medial coxa D & $44( \pm) 3$ & $43( \pm) 2$ & 0.87 \\
Inferior coxa D & $37( \pm) 2$ & $36( \pm) 0$ & 0.76 \\
Superior coxa E & $53( \pm) 4$ & $52( \pm) 3$ & 0.36 \\
Medial coxa E & $44( \pm) 3$ & $43( \pm) 2$ & 0.88 \\
Inferior coxa E & $37( \pm) 2$ & $37( \pm) 2$ & 0.65 \\
+Dobras cutâneas & $47( \pm) 17$ & $50( \pm) 10$ & 0.60 \\
\% Gord. DC & $22( \pm) 5$ & $23( \pm) 3$ & 0.58 \\
Peso (cm) & $56( \pm) 6$ & $56( \pm) 7$ & 0.89 \\
IMC (kg/m²) & $21( \pm) 2$ & $21( \pm) 2$ & 0.85 \\
Nível satisfação & $6( \pm) 1$ & $7( \pm) 2$ & 0.65 \\
pessoal & & & \\
Gordura (\%) & $24( \pm) 3$ & $26( \pm) 3$ & 0.30 \\
Massa magra (\%) & $43( \pm) 4$ & $42( \pm) 5$ & 0.57 \\
Água (\%) & $53( \pm) 3$ & $53( \pm) 3$ & 0.64 \\
Impedância & $676( \pm) 59$ & $710( \pm) 38$ & 0.97 \\
\hline
\end{tabular}

Legenda: Os valores representam a média ( \pm desvio-padrão). Fonte: Dados da pesquisa.

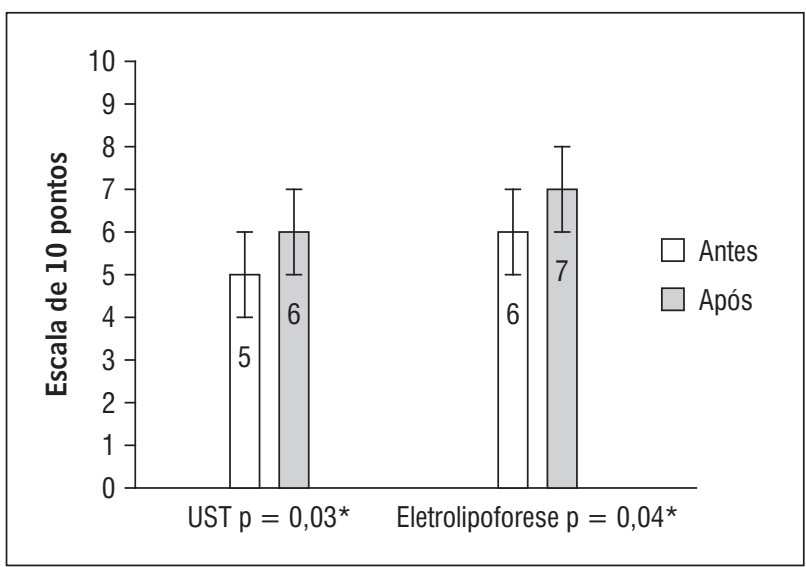

Figura 2 - Comparação dos valores médios de satisfação antes e após os tratamentos realizados

\section{Discussão}

Francischelli Neto (2) escreveu que $87 \%$ das pacientes com FEG apresentavam flacidez muscular, decorrente do sedentarismo, e a maioria dessas pacientes apresentaram também gordura corporal total ou regional aumentada, caracterizando a SDC.

Neste estudo, as voluntárias apresentaram IMC dentro da normalidade, assim como a média do RCQ também ideal para mulheres. Isso retrata homogeneidade dos grupos estudados, porém observou-se elevado percentual de gordura corporal utilizando-se a adipometria e a bioimpedância. 0 mesmo foi observado por Francischelli, Junqueira e Francischelli (24), que demonstraram que tanto o IMC quanto a RCQ e as medidas absolutas de cintura são eficientes como indicadores de saúde, mas não para determinar a gordura corporal total. Da mesma forma, para Yao et al. (25), o IMC demonstrou baixa sensibilidade na identificação do excesso de gordura corporal, ocorrendo também o mesmo no estudo de Carrasco et al. (26), utilizando-se a bioimpedância. A população com FEG, embora possa apresentar peso e IMC dentro dos padrões normais, apresenta excesso de gordura localizada, detectada por exames que determinam compartimentos, como a bioimpedância e a ressonância magnética.

No presente estudo, analisando os valores médios do peso corporal, IMC e RCQ antes e após os tratamentos instituídos, não houve diferença estatisticamente significante entre eles. Isso sugere que os recursos eletrofísicos utilizados podem desencadear apenas efeitos localizados e discretos na região alvo, o que vem de encontro com os estudos de Paula, Pichet, Simões (14), em que a aplicação da eletrolipólise revelou redução perimétrica significativa na região tratada, mas não alterou os valores de peso e IMC. Assim como no estudo de caso de Garcia, Garcia e Borges (27), em que a aplicação de 18 sessões de eletrolipólise em flancos duas vezes na semana seguida de caminhada resultou em redução do tecido celular subcutâneo adiposo na região tratada em 19,86\%, por meio da ressonância nuclear magnética. Scorza et al. (28) também observaram redução do perímetro abdominal após dez sessões de eletrolipólise em 16 voluntárias sedentárias, por meio do exame ultrassonográfico.

A avaliação das dobras cutâneas também não apresentou diferença estatisticamente significante neste estudo, pela não interferência na camada de gordura nos grupos tratados e pela aplicação dos recursos eletrofísicos estar localizada em um único segmento corpóreo. Nenhuma voluntária do estudo apresentou dor ou alteração da sensibilidade na avaliação inicial, estando de acordo com a literatura, pois os graus iniciais de FEG (I e II) ainda não apresentam nódulos volumosos e de consistência muito 
firme capazes de comprimir nervos e vasos na parede conjuntiva $(17,18,29,30)$.

A satisfação das voluntárias quanto ao tratamento aplicado obteve significância estatística em ambos os grupos, indicando melhora do aspecto visual do FEG, porém, graças ao pequeno número de sessões, esses efeitos não atingiram a máxima satisfação relacionada com a redução de medidas.

Utilizando a interpretação fotográfica e aplicando as bases apropriadas de fotointerpretação, foi possível analisar os resultados obtidos com os diferentes métodos eletrofísicos no aspecto do FEG. As fotografias digitais têm sido utilizadas para capturar e documentar as amplas manifestações de doenças da pele, pois permitem a comunicação entre terapeutas e o paciente (31). Neste estudo houve alta concordância entre os avaliadores quanto à análise do acometimento do FEG por meio das imagens fotográficas, onde indicaram melhora significante da aparência do FEG em ambos os grupos. Isso indica que as aplicações tanto do UST quanto da eletrolipólise foram eficazes para melhorar o aspecto visual do FEG.

Com a análise dos resultados obtidos após a aplicação de dez sessões tanto do UST como da eletrolipólise, não houve significância estatística para a redução das medidas antropométricas e bioimpedância, obtendo-se resultado estatisticamente significante apenas na satisfação pessoal e melhora da aparência do FEG. Isso indica que os efeitos terapêuticos foram localizados e discretos.

Contudo, o uso da estimulação elétrica pode ser útil nos casos de SDC por apresentar melhora no tônus muscular, diminuir massa gorda e melhorar o aspecto do FEG (24), sendo necessários protocolos de tratamento com um número maior de sessões para verificar a eficácia dessa modalidade na estética corporal.

É importante lembrar que, para um tratamento mais eficaz, que recupere a perfeita harmonia corporal, é imprescindível uma terapia combinada com diferentes recursos terapêuticos, exercícios físicos e reeducação de hábitos alimentares, não avaliados neste estudo, que objetivou somente analisar as respostas localizadas desencadeadas pelos recursos eletrofísicos utilizados.

\section{Conclusão}

A partir dos resultados obtidos neste estudo, conclui-se que a aplicação tanto do UST como da eletro- lipoforese apresentou resultados semelhantes, obtendo-se considerável efeito na melhora do aspecto do FEG bem como da satisfação pessoal das voluntárias, porém as demais variáveis, como perimetria, adipometria e bioimpedância, não apresentaram diferença estatisticamente significante.

Assim, tornam-se necessários outros estudos que permitam analisar a eficácia dos recursos eletrofísicos, com maior número de sessões. É importante ressaltar a intervenção direta de outras terapêuticas, como reeducação alimentar e atividade física regular, nas variáveis utilizadas no estudo do FEG.

\section{Referências}

1. Sgreccia E. Manual de bioética: fundamentos e ética biomédica. São Paulo: Loyola; 1996.

2. Francischelli M, Neto. Estudo da composição corporal e suas implicações no tratamento da hidrolipodistrofia. Rev Med Estét Soc Bras Med Estét. 2003; 15:20-7.

3. Pires de Campos MSM. Fibroedema geloide subcutâneo. Ciência \& Tecnologia. 1992;1(2):77-2.

4. Querleux B, Cornillon C, Jolivet O, Bittoun J. Anatomy and physiology of subcutaneous adipose tissue by in vivo magnetic resonance imaging and spectroscopy: Relationships with sex and presence of cellulite. Skin Res Technol. 2002;8(2):118-24.

5. Mirrashed F, Sharp JC, Krause V, Morgan J, Tomanek B. Pilot study of dermal and subcutaneous fat structures by MRI in individuals who differ in gener, BMI, and cellulite grading. Skin Res Technol. 2004;10(3): 161-8.

6. Terranova F, Berardesca E, Maibach I. Cellulite: nature and aetiopathogenesis. Int J Cosmet Sci. 2006; 28(3):157-67.

7. Rosenbaum M, Prieto V, Hellmer J, Boschmann M, Krueger J, Leibel RL, et al. An exploratory investigation of the morphology and biochemistry of cellulite. Plast Reconstr Surg. 1998;101(7):1934-9.

8. Alaniz MHF, Takada J, Vale MICA, Lima FB. 0 tecido adiposo como centro regulador do metabolismo. Arq Bras Endocrinol Metab. 2006;50(2):216-29.

9. Quatressoz P, Ulhoa EX, Franchimont CP, Piérard GE. Cellulite histopathology and related mechanobiology. Int J Cosmet Sci. 2006;28(3):207-10. 
10. Rawlings AV. Cellulite and its treatment. Int J Cosmet Sci. 2006;28(3):175-90.

11. Draelos ZD, Marenus KD. Cellulite: etiology and purported treatment. Dermatol Surg. 1997;23(12): 1177-81.

12. Angelino BS. Fibroedema geloide subcutâneo: qué conocemos de esta entidad clínica? Folia Dermatol Peru. 2003;14(1):38-42.

13. Durigan JLQ, Cancelliero KM, Reis MS, Dias CNK, Graciotto DR, da Silva CA, et al. Mecanismos de interação do ultra-som terapêutico com tecidos biológicos. Rev Fisioter Brasil. 2006;7(2):142-8.

14. de Paula MR, Picheth G, Simões NP. Efeitos da eletrolipoforese nas concentrações séricas do glicerol e do perfil lipídico. Fisioter Brasil. 2007;8(1):5-9.

15. Braz, GP. Sistema de eletroestimulação informatizado para tratamento da dor e para a reabilitação neuromuscular. [dissertação]. Florianópolis: Universidade Federal de Santa Catarina; 2003.

16. de Macedo ACB, Simões ND. Aplicação de estimulação elétrica de baixa intensidade no tratamento de úlceras varicosas. Fisioter Mov. 2007;20(3):25-33.

17. Meyer PF, Lisboa FL, Alves MCR, Avelino MB, et al. Desenvolvimento e aplicação de um protocolo de avaliação fisioterapêutica em pacientes com fibro edema geloide. Fisioter Mov. 2005; 18(1):75-83.

18. Guirro ECO, Guirro RRJ. Fisioterapia dermato-funcional: fundamentos, recursos e patologias. São Paulo: Manole; 2006.

19. Glaner MF. Índice de massa corporal como indicativo da gordura corporal comparado às dobras cutâneas. Rev Bras Med Esporte. 2005;11(4):243-6.

20. Guedes DP. Recursos antropométricos para análise da composição corporal. Rev Bras Educ Física. 2006; $20: 115-9$

21. Rona C, Carrera M, Berardesca E. Testing anticellulite products. Int J Cosmet Sci. 2006;28(3):169-73.

22. Fett CA, Fett WCR, Marchini JS. Comparação entre bioimpedância e antropometria e a relação de índices corporais ao gasto energético de repouso e marcadores bioquímicos sanguíneos em mulheres da normalidade à obesidade. Rev Bras Cineantropom Desempenho Hum. 2006;8(1):29-36.
23. Ribeiro JP, Cummins R. O bem estar pessoal: estudo da validação da versão portuguesa da escala. Actas do 7ํㅡ Congresso Nacional de Psicologia da Saúde. Lisboa: ISPA; 2008. p. 505-8.

24. Francischelli M, Neto, Francischelli RT, Junqueira LOR. Hidrolipodistrofia: avaliação epidemiológica e uma proposta de classificação. Rev Med Estét Soc Bras Med Estét. 2001;12:27-36.

25. Yao M, Roberts SB, Ma G, Pan H, Mccrory MA. Field methods for body composition assessment are valid in healthy Chinese adults. J Nutr. 2002;132(2):310-7.

26. Carrasco F, Reyes E, Rimier 0, Rios F. Exactitud del índice de masa corporal en la predicción de la adiposidad medida por impedanciometria bioeléctrica. Arq Latino-americanos de Nutrição. 2004;54(3):280-6.

27. Garcia PG, Garcia FG, Borges FS. 0 uso da eletrolipólise na correção da assimetria no contorno corporal póslipoaspiração: relato de caso. Rev Fisioter Ser. 2006; 1(4):287-92.

28. Scorza FA, Figueiredo MM, Liao CO, Borges FS. Estudo comparativo dos efeitos da eletrolipólise com uso de tens modo burst e modo normal no tratamento de adiposidade localizada abdominal. Rev Ensaios e Ciência: Ciênc Biológ Agrárias e da Saúde. 2008; 12(2):49-62.

29. Peña J, Pérez MH. Lipodistrofia ginecoide (celulitis). Rev Centro Dermatológico Pascua. 2005;14(3):132-5.

30. Angelino BS. Fibroedema geloide subcutâneo: qué conocemos de esta entidad clínica? Folia Dermatol Peru. 14(1):38-42.

31. Baraúna MA, Ricieri D. Biofotogrametria: recurso diagnóstico do fisioterapeuta. [acesso em: 30 mar. 2007]. Disponível em: http://www.coffito.org.br

Recebido: $11 / 11 / 2010$

Received: $11 / 11 / 2010$

Aprovado: 23/07/2011

Approved: 07/23/2011 